\title{
DETERMINACIÓN DE LA BASE REGIONAL DE RECURSOS LÍTICOS DEL ÁREA RÍO MANSO INFERIOR-FOYEL (RÍO NEGRO). PRIMEROS RESULTADOS
}

\author{
Mariana Carballido Calatayud ${ }^{1}$ y Fernando Xavier Pereyra ${ }^{2}$ \\ ${ }^{1}$ CONICET-INAPL-UBA 3 de febrero 1378, C.A.B.A., Argentina. E-mail: mcarballidocalata@hotmail.com \\ ${ }^{2}$ SEGEMAR Av. Presidente J. A. Roca 651, C.A.B.A., Argentina. E-mail: fernap@mecon.gov.ar
}

Presentado el: 23/12/2011 - Aceptado 24/03/2012

\section{Introducción}

Las investigaciones que se vienen desarrollando en el bosque del suroeste de Río Negro y noroeste de Chubut han expuesto los desafíos que implica habitar este ambiente para sociedades que basan su subsistencia en la caza y recolección (Bellelli et al. 2000, Carballido Calatayud 2009, Fernández 2008). Desarrollar esta forma de vida en los bosques presenta mayores dificultades que en la estepa, ya que en ellos hay una menor biomasa animal y las potenciales presas frecuentemente son mamíferos de porte mediano a pequeño, de hábitos solitarios y dispersos. Además, el desarrollo de la vegetación dificulta el tránsito y la visibilidad de los recursos dentro del bosque (Carballido Calatayud 2009, Fernández et al. 2011a, Scheinsohn 2004). En general existe cierto consenso en que la ocupación de este ambiente implicó algún tipo de complementariedad ambiental (Arrigoni 1997, Bellelli et al. 2003, Lezcano et al. 2010, Hajduk et al. 2004, Silveira 1999). En relación con las materias primas líticas para la talla, la existencia de dicha complementariedad o más precisamente la presencia de rocas no locales en los conjuntos arqueológicos de bosque ha sido informada por diversos autores. En algunos casos estas rocas constituyen la totalidad o casi la totalidad del material identificado (Hajduk et al. 2004) mientras que en otros la proporción de materias primas locales y no locales es variable (López 2010, Silveira 1996). Para ambas situaciones, la disponibilidad local y en algunos casos la abundancia de rocas ha sido considerada como el principal factor para explicar la representación de las materias primas en los conjuntos líticos (Hajduk et al. 2004, López 2010, Silveira 1996).

En trabajos previos en el área boscosa de Cholila (Chubut) planteamos que además de la disponibilidad y abundancia de rocas, la accesibilidad y la visibilidad eran factores igualmente determinantes (Carballido Calatayud 2009, 2011). La importante cobertura vegetal que caracteriza a los bosques se traduce en una baja visibilidad de las potenciales fuentes de materias primas líticas y restringe los desplazamientos disminuyendo la accesibilidad a las canteras. A la vez, la dinámica de los incendios forestales, que a lo largo del tiempo modifica la fisonomía del bosque, habría dificultado la reubicación de las fuentes luego de 
determinado lapso, dejando también al descubierto nuevas canteras potenciales (Carballido Calatayud 2009, 2011). Para superar las dificultades de la localización y reubicación de las materias primas líticas en el bosque propusimos dos comportamientos que podrían incluso ser complementarios: el aprovisionamiento en fuentes del ecotono o de la estepa y/o el uso de las materias primas locales más fáciles de reubicar, como las emplazadas en las playas de ríos, lagos o lagunas. Los resultados alcanzados en Cholila muestran la disponibilidad y abundancia de basaltos, calcedonias y en menor cantidad, de sílices. En todos los casos estas rocas fueron localizadas en playas o en sectores con vegetación menos densa. Por otra parte, estas materias primas constituyen una proporción importante de los conjuntos artefactuales, que están compuestos por una elevada proporción de instrumentos descartados al final de su vida útil y, muchos de ellos, con evidencias de un uso económico o maximización de las rocas (Bamforth 1986, Bayón et al. 1995, Shott 1989). Pudimos ver entonces que una parte de las materias primas locales se trataron cuidadosamente, sin derroche tal como se esperaría para rocas no locales o poco disponibles y/o escasas. Así, en este caso la disponibilidad y abundancia local parecerían no alcanzar para explicar el tratamiento dado a los conjuntos artefactuales. Una explicación alternativa sería que las dificultades en la accesibilidad y visibilidad, que elevan los costos de búsqueda y relocalización, habrían llevado a optimizar las rocas halladas (Carballido Calatayud 2009, 2011).

La variabilidad de situaciones expuestas en relación con las modalidades de aprovechamiento de materias primas líticas en el bosque podría vincularse en parte a la distancia y la accesibilidad a zonas ecotonales o de estepa en las que, entre otros factores, la visibilidad y accesibilidad a las fuentes podría ser mayor. Es por ello que consideramos la necesidad de generar modelos locales que tengan en cuenta estos factores. Así, nos hemos propuesto explorar en qué medida el emplazamiento hacia el interior del bosque mixto de Nothofagus y Austrocedrus de Norpatagonia influyó en las decisiones tecnológicas líticas desarrolladas por grupos cazadores-recolectores. El trabajo que aquí presentamos es una primera aproximación al problema ya que su objetivo es presentar los resultados de las prospecciones que tuvieron como fin comenzar a evaluar la base regional de recursos líticos (Ericson 1984).

\section{El caso de estudio}

El área de investigación abarca los valles de los ríos Manso inferior y Foyel (provincia de Río Negro), aproximadamente entre $71^{\circ} 30^{\prime}$ longitud oeste y la frontera con Chile y se ubica inmediatamente al sur del límite del Parque Nacional Nahuel Huapi (Figura 1). Comprende un ambiente de bosque dominado por distintas especies de Nothofagus que también presenta extensas formaciones de Austrocedrus chilensis, sobre todo en sectores perturbados y en los faldeos. A medida que se avanza hacia la cordillera la vegetación se hace más densa ya que comienzan a aparecer especies típicas del bosque lluvioso (Armesto et al. 1995; Podestá et al. 2008). En el área se han registrado 21 sitios arqueológicos, todos con manifestaciones rupestres. La mayoría se localiza en los valles fluviales aunque algunos están en los faldeos de los cerros que rodean a los valles. Se trata de aleros, paredes rocosas y bloques, a los que se suman varios objetos aislados hallados por pobladores como manos, molinos y un toki (Fernández et al. 2011b). Este sector de bosque registra ocupaciones desde hace 3400 años, registrándose un incremento en la señal arqueológica a partir de 1600 años A. P. (Fernández et al. 2011b). Hasta el momento se han excavado cinco de estos sitios y el material lítico proveniente de dos de ellos (Paredón Lanfré y Población Anticura) está bajo análisis. 


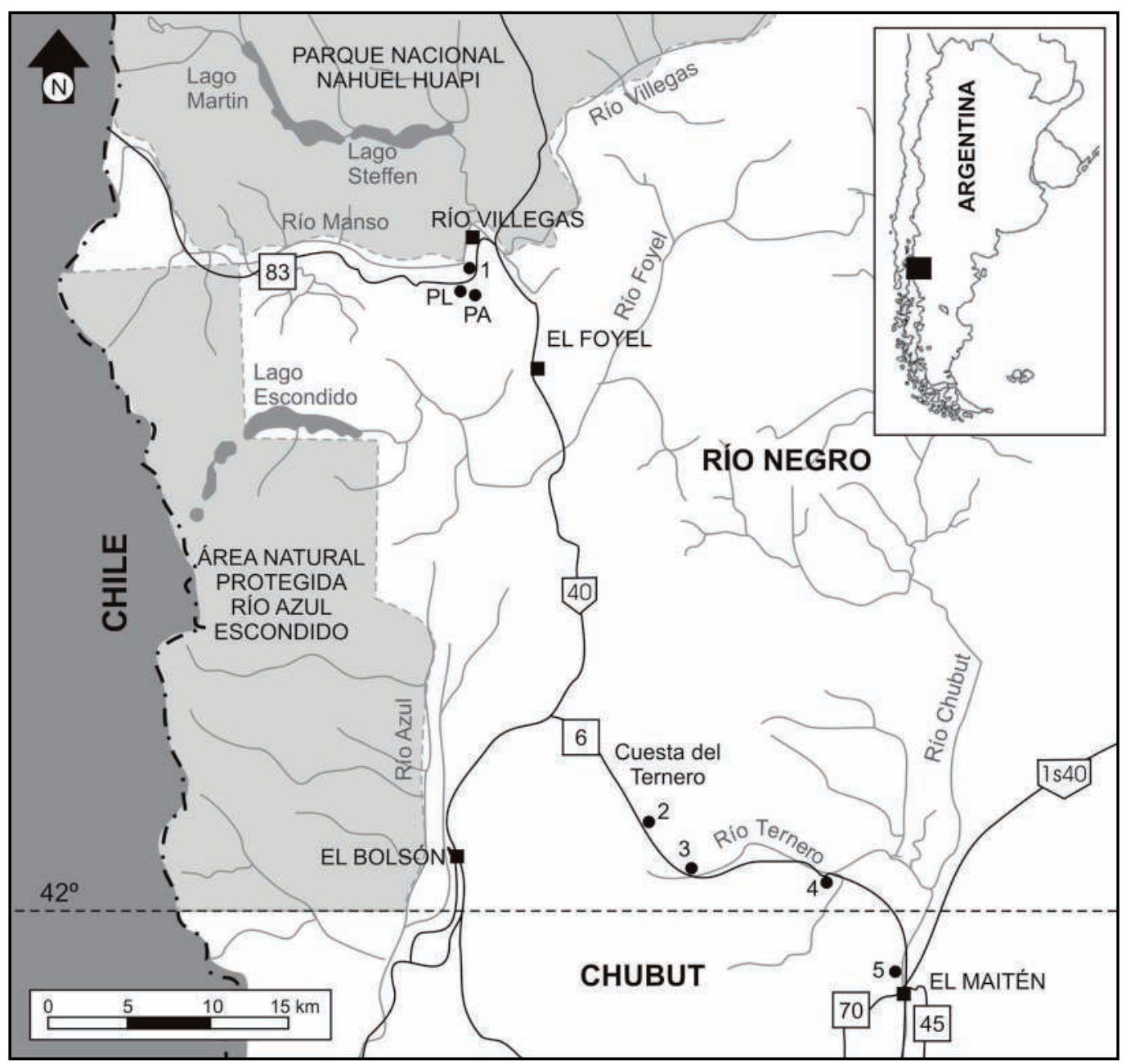

Figura 1. Mapa de ubicación de los sitios y puntos de muestreo mencionados en el texto. Referencias: PL: Paredón Lanfré, PA: Población Anticura.

La oferta de rocas potencialmente utilizables como materias primas es variada en toda la región (Figura 2). Desde el punto de vista geológico, ésta corresponde a una faja plegada y corrida de retroarco formada como consecuencia de la Orogenia Andina. Este proceso comenzó hace al menos 60 Ma y continua activo. Consecuentemente, la estructura presenta una orientación norte-sur constituyendo una serie de láminas de corrimiento, generalmente con vergencia al este que implican variadas litologías. Entre ellas destacan, en la zona de la Cuesta del Ternero, pórfiros graníticos y riolíticos y metamorfitas de bajo grado correspondientes a un antiguo Plutón paleozoico y volcanitas y piroclastitas del Terciario Inferior de la Formación Ventana. Hacia el oeste aparecen también sedimentitas y volcanitas juráricas del Complejo Volcánico-sedimentario Jurásico, así como sedimentitas y piroclastitas neógenas y granitoides terciarios. Finalmente, tanto en la zona del límite como hacia el este, se encuentran volcanitas (andesitas y basaltos) pliocenos y pleistocenos (Giacossa et al. 2001). 


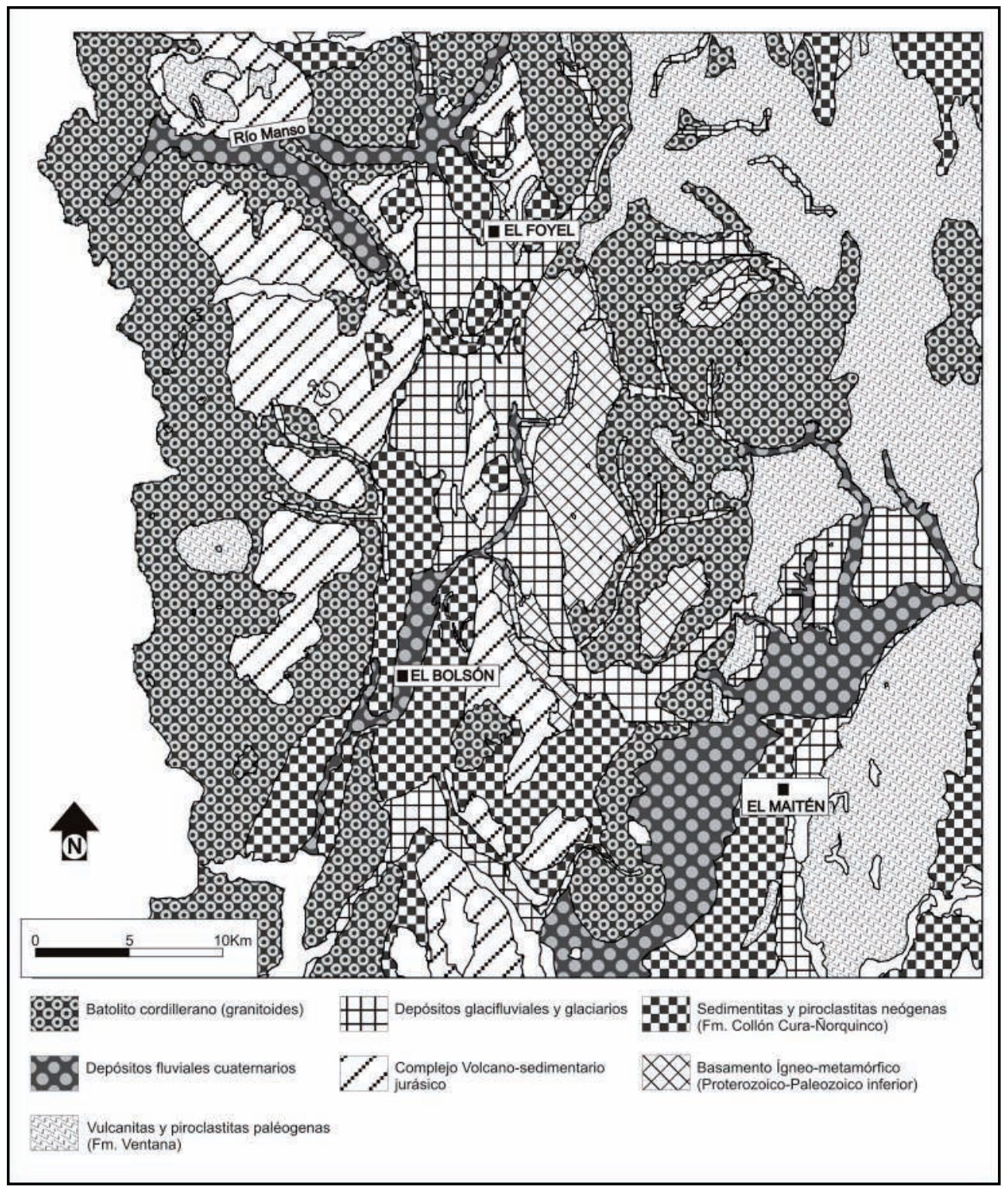

Figura 2. Mapa litológico del área de investigación. Tomado de las Cartas de Peligrosidad Geológica, Cartas Litológicas de San Carlos de Bariloche 4172-IV (Wilson et al. 2007) y de Esquel 4372-II y I (Chavez et al. 2009). Escala 1:250.000.

En relación a la geomorfología, la zona muestra la evidencia de un englazamiento total, salvo en los sectores más elevados. Evidencias de al menos dos Glaciaciones son reconocibles en la región, así como rasgos glaciales más modernos correspondientes al Tardiglacial y Neoglacial. En al zona de la Cuesta del Ternero una lengua glaciaria se proyectó desde el valle de El Bolsón-Mallín Ahogado hacia el este convergiendo con una gran lengua que venía desde el sur, por el valle del Maitén. Estos glaciares dieron como resultado una serie de cordones morénicos bien representados al NE de ese valle (hacia Norquinco). Con el 


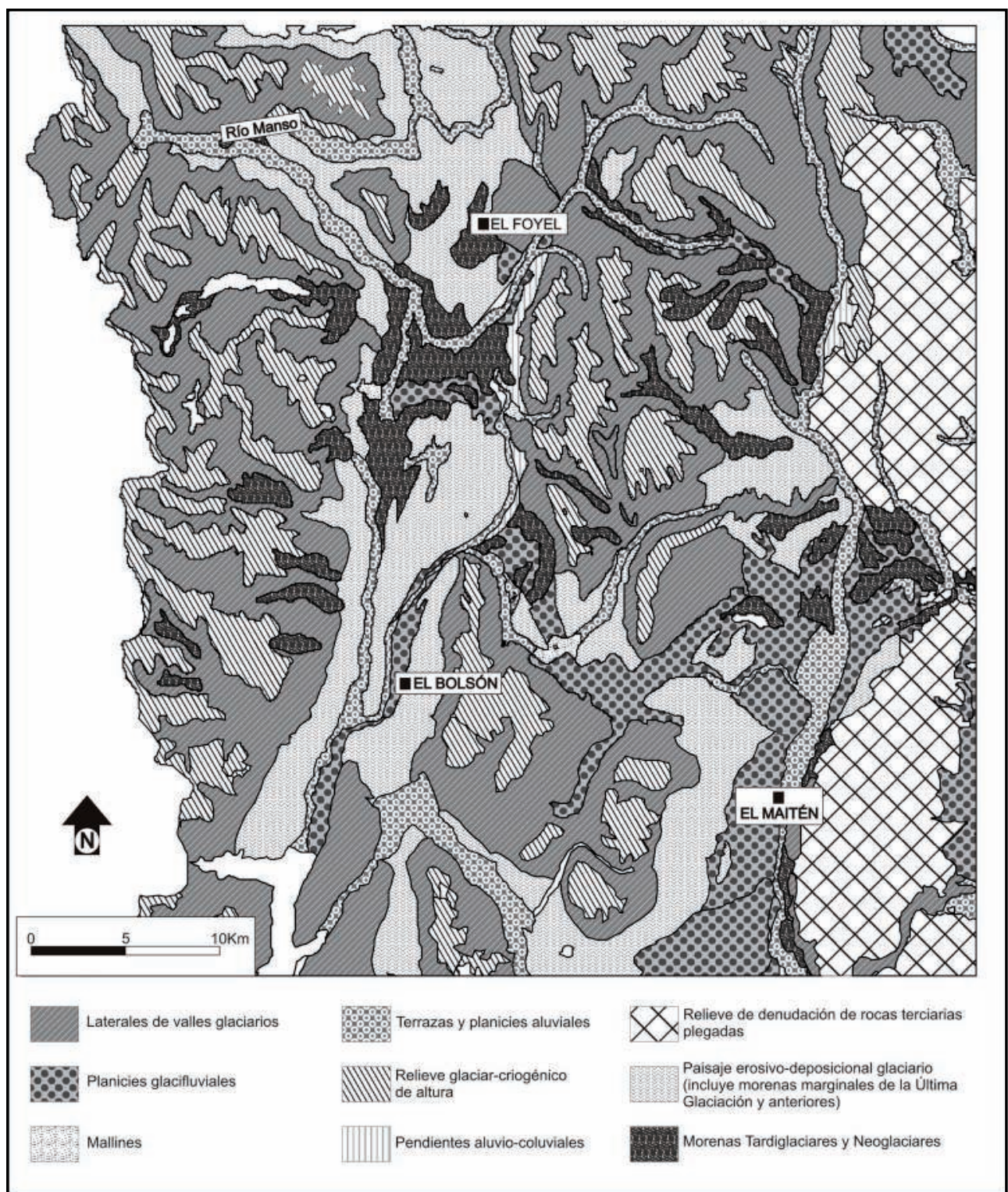

Figura 3. Mapa geomorfológico del área de investigación. Tomado de las Cartas de Peligrosidad Geológica, Cartas Geomorfológicas de San Carlos de Bariloche 4172-IV (Pereyra et al. 2007) y de Esquel 4372-II y I (Tejedo y Pereyra 2009). Escala 1:250.000.

retroceso de los glaciares, ambas lenguas se desvincularon y la proveniente del oeste dejó como registro las morenas ubicadas en la zona de la Cuesta del Ternero. Al frente de las mismas se desarrollaron planicies glacifluviales que se extienden hasta el actual valle del río Chubut. Es posible identificar además algunos abanicos aluviales más modernos que conforman un nivel de bajada que ha progradado sobre las planicies glacifluviales y algunos pequeños remanentes de niveles de pedimentos (Figura 3). Tanto las planicies glacifluviales como las bajadas, pedimentos y terrazas fluviales del río Chubut pueden constituir importantes fuentes potenciales de aprovisionamiento (Flint y Fidalgo 1964). 


\section{Relevamientos}

Los trabajos de relevamiento orientados a reconocer fuentes de materias primas potencial o efectivamente utilizadas consistieron en prospecciones asistemáticas guiadas por la información geológica generada por uno de nosotros y obtenida a partir de la Hoja Geológica Bariloche (Giacossa et al. 2001) y recorridas basadas en datos proporcionados por pobladores locales. El primer espacio elegido para ser relevado fue un sector de la playa del río Manso (Punto 1, Figura 1) que se encuentra ca. 2,5 km lineales del sitio Paredón Lanfré. Este río posee sectores en los que la sedimentación ha cubierto los guijarros de las playas y otros en los que éstos están expuestos. Según datos de pobladores uno de estos sectores de playa compuesta de guijarros podía presentar algunas rocas que se encuentran en los conjuntos arqueológicos, por ejemplo riolitas y andesitas de grano fino. En el relevamiento realizado hallamos guijarros de andesitas de entre 140 y $40 \mathrm{~mm}$ de diámetro y con distintos tamaños de grano, algunos aptos para la talla por su buena fractura. También identificamos un guijarro de una riodacita de $110 \mathrm{~mm}$ de diámetro que tiene una venilla de cuarzo de 50 $\mathrm{mm}$ de espesor y otros de tobas vítreas de entre 90 y $50 \mathrm{~mm}$ de diámetro de grano muy fino $\mathrm{y}$ fractura concoidea.

En segundo lugar, decidimos privilegiar la prospección de las planicies glacifluviales en las que estuvieran expuestos los depósitos de rocas. A partir del estudio de la Hoja Geológica Bariloche (Giacossa et al. 2001) y de la experiencia de campo directa de uno de nosotros se identificaron una serie de espacios potencialmente interesantes. Uno de ellos son los glacifluviales que se encuentran en el camino de la Cuesta del Ternero, que conecta la zona de El Bolsón y Mallín Ahogado con la localidad de El Maitén. Recorrimos ca. 28 kilómetros lineales haciéndose los relevamientos en los sectores en los que los depósitos glacifluviales estaban expuestos o en otros en los que hubiera bloques u afloramientos. El primer lugar que revisamos se encuentra ca. $38 \mathrm{~km}$ del sitio Paredón Lanfré (Punto 2, Figura 1). Se trata de un sector con bloques de arrastre glacial principalmente de granito y de andesitas de grano grueso, no aptas para la talla. Allí detectamos la presencia de una calcedonia en forma de un guijarro de unos $75 \mathrm{~mm}$ de diámetro, de fractura angular o plana y calidad regular para la talla. El segundo lugar está a $3 \mathrm{~km}$ al sureste del punto anterior (Punto 3, Figura 1). Corresponde a un depósito glacifluvial relacionado con el curso del río Ternero, que corre a unos 35 - 40 metros de distancia. Revisamos extensas acumulaciones de guijarros de diversos tamaños y localizamos granitos, rocas volcánicas -principalmente andesitas- y en menor frecuencia brecha volcánica y calcedonia. Algunas de las andesitas son de grano fino y fractura concoidea, siendo de calidad regular a buena. Se presentan en guijarros de entre 70 y $110 \mathrm{~mm}$ de diámetro. La calcedonia detectada es de características similares a las vistas en el primer sector revisado. Por último, la brecha volcánica también se presenta en forma de guijarros, algunos de tamaños menores (40 a $50 \mathrm{~mm}$ de diámetro) y con una mala calidad para la talla a raíz de la presencia de inclusiones que dirigen las fracturas planas. También relevamos un sector de bloques de derrumbe de una ladera a ca. $11 \mathrm{~km}$ del punto anterior (Punto 4, Figura 1). Allí registramos la presencia cuarzo y de rocas volcánicas: andesitas, dacitas, riodacitas, de una brecha volcánica similar a la vista anteriormente. La andesita es la que posee mejor calidad para la talla, es de grano fino y tiene fractura concoidea. Se presenta en bloques medianos a pequeños (de unos 150 a 200 $\mathrm{mm}$ de diámetro). Las dacitas y riodacitas, a pesar de ser de grano fino tienen fractura plana por lo que no son aptas para la talla. El cuarzo, incluido en una matriz volcánica y con un espesor de aproximadamente $50 \mathrm{~mm}$ también presenta una fractura angulosa. En la localidad de El Maitén se relevó un sector de la costa del río Chubut, el punto más alejado 
del recorrido, a ca. $58 \mathrm{~km}$ de Paredón Lanfré (Punto 5, Figura 1). Se trata de una playa de guijarros y rodados que son los mismos que componen el lecho del río. Allí se detectaron sobre todo andesitas y basaltos con distintos tamaños de grano y tipos de fractura y con un tamaño promedio de $100 \mathrm{~mm}$. También se hallaron guijarros de brecha volcánica de grano grueso y de una roca volcánica ácida de grano muy fino y fractura concoidea, también de $100 \mathrm{~mm}$ de diámetro. Por último se halló un pequeño guijarro (de $30 \mathrm{~mm}$ de diámetro) de una roca silícea de textura amorfa y fractura concoidea.

\section{Palabras finales}

Esta primera aproximación a la base regional de recursos líticos contempló el relevamiento de espacios cercanos a los principales sitios y de otros más alejados. Entre los primeros priorizamos un sector de la playa del río Manso inferior, con el objetivo de evaluar si estos espacios, más accesibles y relativamente más fáciles de reubicar, presentaban rocas aptas para la talla. Los resultados alcanzados muestran que constituyen áreas potenciales de aprovisionamiento, sobre todo de rocas volcánicas. Por su parte, los sectores más alejados están representados por el camino denominado Cuesta de Ternero, seleccionado para su relevamiento por la variada oferta de potenciales materias primas y porque constituye una de las vías de acceso más transitables -a esta latitud- que conecta el bosque con el ecotono y la estepa y permite contemplar la problemática de la complementariedad ambiental a la que hemos hecho referencia al inicio de esta nota. Allí constatamos que están disponibles rocas volcánicas, cuarzo, calcedonia y sílices, cuya disponibilidad podría verse afectada estacionalmente por la mayor carga nival que recibe esta área más elevada $(700-760 \mathrm{msnm})$ que el valle del Manso (490 msnm).

La información reseñada aquí es la base para continuar los relevamientos en otros sectores. Además, junto con la generada a partir de los análisis líticos brindará un panorama más acabado de las decisiones de manejo y uso de materias primas líticas que se llevaron a cabo en el pasado en esta zona del interior del bosque de Norpatagonia.

\section{Agradecimientos}

Estamos en deuda con varias personas, principalmente con Soledad Caracotche quien colaboró activamente en los trabajos de relevamiento, con Pablo Fernández por la lectura del manuscrito y con Ana Forlano por su ayuda en la confección de la figura 1. Agradecemos a todo el equipo de investigación por el apoyo de siempre, muy especialmente a Pedro Fernández Carballido y su abuela Didi por su ayuda en el relevamiento de la playa del río Manso y a Manuel Fernández Carballido que desde la panza acompañó en todas las prospecciones. También estamos en deuda con el evaluador del manuscrito por sus comentarios y sugerencias. Este trabajo fue financiado por los proyectos PICT ANPCyT 26332 y UBACYT U013.

\section{Bibliografía citada}

\section{Arrigoni G. I.}

1997. Pintando entre bosques y lagos. Actas y Memorias del XI Congreso Nacional de Arqueología Argentina, Primera Parte, Revista del Museo de Historia Natural de San Rafael Tomo XIII No $1 / 4:$ 58-63.

Armesto, J. J., P. León Lobos y M. Kalin Arroyo

1995. Los Bosques templados del sur de Chile y Argentina: una isla biogeográfica. En Ecología de los bosques nativos de Chile, (ed. por J. J. Armesto, C. Villagrán y M. Kalin Arroyo), pp. 23-28 Editorial Universitaria, Santiago de Chile. 
Bamforth, D. B.

1986. Technological Efficiency and Tool Curation. American Antiquity 51(1):38-50.

Bayón, C., P. Escola y N. Flegenheimer

1995. Organización tecnológica: usos y abusos de esta perspectiva. Arqueología 5: 179-186.

Bellelli, C., V. Scheinsohn, P. Fernández, F. Pereyra, M. Podestá y M. Carballido

2000. Arqueología de la Comarca Andina del Paralelo $42^{\circ}$. Localidad de Cholila. Primeros resultados. En Desde el País de los Gigantes. Perspectivas arqueológicas en Patagonia, pp. 587-602. Universidad Nacional de la Patagonia Austral, Río Gallegos.

Bellelli, C., M. Carballido, P. Fernández y V. Scheinsohn 2003. El pasado entre las hojas. Nueva información arqueológica del noroeste de la provincia de Chubut, Argentina. Revista Werken 4: 25-42.

Carballido Calatayud, M.

2009. Organización de la tecnología lítica en el bosque de Norpatagonia durante el Holoceno tardío. Aportes para un modelo de uso del bosque en la Comarca Andina del Paralelo $42^{\circ}$. Tesis de Doctorado en Arqueología, Facultad de Filosofía y Letras, Universidad de Buenos Aires. Ms.

2011. Agujas en un pajar: el aprovisionamiento lítico en el bosque desde la perspectiva de la localidad de Cholila (Chubut). Trabajo presentado en las VIII Jornadas de Arqueología de la Patagonia, 3 al 7 de octubre de 2011, Malargüe, Mendoza. Ms.

Chavez, R., A. Tejedo y N. Tello

2009. Carta de Peligrosidad Geológica. Esquel. 4372-II y I. Carta Litológica. Escala 1:250.000. Servicio Geológico Minero Argentino, Instituto de Geología y Recursos Minerales, Dirección de Geología Ambiental Aplicada. Buenos Aires.

Ericson, J. E.

1984. Toward the analysis of lithic reduction systems. En Prehistoric Quarries and Lithic Production (ed. por J. E. Ericson y B. Purdy), pp. 11-22. Cambridge University Press, Cambridge.

Fernández, P. M.

2008. Taphonomy and zooarcheology in the Neotropics: a view from northwestern Patagonian forest and steppe. Quaternary International 180: 63-74.

Fernández, P., M. Carballido Calatayud, C. Bellelli, M. Podestá y V. Scheinsohn 2011a. Marcas en la piedra, huellas en la tierra. El poblamiento del bosque del suroeste de Río Negro- noroeste de Chubut. En Procesos históricos, transformaciones sociales y construcciones de fronteras. Aproximaciones a las relaciones interétnicas. Estudios sobre Norpatagonia, Argentina y Labrador, Canadá (Coord. por S. Valverde, G. Maragliano, M. Impemba y F. Trentini), pp. 195-221 Editorial de la Facultad de Filosofía y Letras, Universidad de Buenos Aires, Buenos Aires.

Fernández, P. M., C. Bellelli, M. Carballido Calata y M. M. Podestá

2011b. Tiempo de cazadores. Cronología de las ocupaciones humanas en el valle del río Manso inferior (Río Negro). Trabajo presentado a las VIII Jornadas de Arqueología de la Patagonia, 3 al 7 de octubre de 2011, Malargüe, Mendoza. Ms. 
Flint. R. y F. Fidalgo

1964. Glacial drift in the eastern Argentine Andes between $41^{\circ} 10^{\prime} \mathrm{S}$ y $43^{\circ} 10^{\prime} \mathrm{S}$. Geological Society or America Bulletin 80: 1043-1052.

Giacossa, R., N. Heredia y O. Césari

2001. Hoja Geológica 4172-IV, S. C. de Bariloche. SEGEMAR-IGRM, Boletín 279.

Hajduk A., A. Albornoz y M. J. Lezcano

2004. El Mylodon en el patio de atrás. Informe preliminar sobre los trabajos en el sitio El Trébol, ejido urbano de San Carlos de Bariloche, provincia de Río Negro. En Contra viento y marea. Arqueología de Patagonia (Comp. por M. T. Civalero, P. M. Fernández y A. G. Guráieb), pp. 715-731. Instituto Nacional de Antropología y Pensamiento Latinoamericano-Sociedad Argentina de Antropología, Buenos Aires.

Lezcano, M. J., A. Hajduk y A. M. Albornoz.

2010. El menú a la carta en el bosque ¿entrada o plato fuerte?: una perspectiva comparada desde la zooarqueología del sitio El Trébol (lago Nahuel Huapi, Pcia. de Río Negro). En Zooarqueología a principios del siglo XXI: aportes teóricos, metodológicos y casos de estudio (Ed. por M. A. Gutiérrez, M. De Nigris, P. M. Fernández, M. Giardina, A. F. Gil, A. Izeta, G. Neme y H. D. Yacobaccio), pp. 243-257. Ediciones del Espinillo, Buenos Aires.

López, L.

2010. Aprovisionamiento y uso de los recursos líticos en el lago Meliquina, bosque meridional neuquino, noroeste de la Patagonia. Tesis de Licenciatura en Ciencias Antropológicas, Facultad de Filosofía y Letras, Universidad de Buenos Aires. Ms.

Podestá, M. M., C. Bellelli, R. Labarca, A. M. Albornoz, A. Vasini y E. Tropea

2008. Arte rupestre en pasos cordilleranos del bosque andino patagónico (El Manso, Región de los Lagos y Provincia de Río Negro, Chile-Argentina). Magallania Vol. 36(2):143-153.

Pereyra, F. X. , D. Roverano y M. Elissondo

2007. Carta de Peligrosidad Geológica. San Carlos de Bariloche. 4172-IV. Carta Geomorfológica. Escala 1:250.000. Servicio Geológico Minero Argentino, Instituto de Geología y Recursos Minerales, Dirección de Geología Ambiental Aplicada. Buenos Aires.

Scheinsohn, V.

2004. "En el país de los ciegos, el tuerto es rey". Visibilidad arqueológica y paisaje en la localidad Cholila. En Contra Viento y Marea. Arqueología de Patagonia, (Comp. por M. T. Civalero, P. M. Fernández y A. G. Guráieb), pp. 581-590. Sociedad Argentina de Antropología e Instituto Nacional de Antropología y Pensamiento Latinoamericano, Buenos Aires.

Shott, M.

1989. On Tool-Class Use Lives and the Formation of Archaeological Assemblages. American Antiquity 54 (1): 9-30.

Silveira, M.

1996. Alero Los Cipreses (Provincia del Neuquén, República Argentina). Arqueología. Sólo Patagonia. Ponencias de las Segundas Jornadas de Arqueología de la Patagonia, (Ed. por J. Gómez Otero), pp. 107-118. CENPAT-CONICET, Puerto Madryn. 
1999. El Alero Lariviére: un sitio en el bosque septentrional andino (Departamento Los Lagos, Provincia del Neuquén, Argentina). En Soplando en el viento. Actas de las Terceras Jornadas de Arqueología de la Patagonia, pp. 83-92. Instituto Nacional de Antropología y Pensamiento Latinoamericano y Universidad Nacional del Comahue. Buenos Aires - Neuquén.

Tejedo, A. y F. X. Pereyra

2009. Carta de Peligrosidad Geológica. Esquel. 4372-II y I. Carta Geomorfológica. Escala 1:250.000. Servicio Geológico Minero Argentino, Instituto de Geología y Recursos Minerales, Dirección de Geología Ambiental Aplicada. Buenos Aires.

Wilson, C., M. Elissondo, D. Roverano y F. X. Pereyra 2007. Carta de Peligrosidad Geológica. San Carlos de Bariloche. 4172-IV. Carta de Unidades Litológicas. Escala 1:250.000. Servicio Geológico Minero Argentino, Instituto de Geología y Recursos Minerales, Dirección de Geología Ambiental Aplicada. Buenos Aires. 